\title{
Entamoeba gingivalis - prevalence and correlation with dental caries in children from rural and urban regions of Lublin Province, Eastern Poland
}

\author{
Maria Mielnik-Błaszczak ${ }^{1, A, E-F}$, Jolanta Rzymowskaa,A-C, Artur Michałowski ${ }^{1, A-D,}$ \\ Agnieszka Skawińska-Bednarczyk ${ }^{1, C-D, F}$, Jerzy Błaszczak ${ }^{3, C, E-F}$ \\ ${ }^{1}$ Chair and Department of Paediatric Dentistry, Medical University, Lublin, Poland \\ ${ }^{2}$ Chair and Department of Biology and Genetics, Medical University, Lublin, Poland \\ ${ }^{3}$ Chair and Department of Jaw Orthopaedics, Medical University, Lublin, Poland \\ A - Research concept and design, B - Collection and/or assembly of data, C - Data analysis and interpretation, \\ $D$ - Writing the article, E - Critical revision of the article, F - Final approval of article
}

\begin{abstract}
Mielnik-Błaszczak M, Rzymowska J, Michałowski A, Skawińska-Bednarczyk A, Błaszczak J. Entamoeba gingivalis - prevalence and correlation with dental caries in children from rural and urban regions of Lublin Province, Eastern Poland. Ann Agric Environ Med. $2018 ; 25(4)$ : $656-658$. doi: 10.26444/aaem/80403
\end{abstract}

\begin{abstract}
Introduction. Entamoeba gingivalis, as the name implies. typically lives around the gumline of the teeth in the tartar and gingival pockets of the oral cavity. Pathogenicity of protozoa in the oral cavity is not completely understood. The occurrence in patients with a good state of the immune system usually does not cause any pathological changes. In the presentede survey, an increased incidence of Entamoeba gingivalis in conjunction with pathological changes in the oral cavity was found.

Objectives. The aim of the study was to evaluate the prevalence of Entamoeba gingivalis in children treated in Department of Paediatric Dentistry of the Medical University in Lublin and its correlation with dental caries.

Materials and method. Diagnostic materials in the form of swabs were obtained from 154 children in the age 2-18 years old, treated in the Department of Paediatric Dentistry of the Medical University in Lublin. The main index for epidemiological surveys in oral health - DMFT (decayed, missed, and filled teeth) for permanent teeth and DMFT for deciduous teeth was calculated.

Results. The average number of Entamoeba gingivalis in urban children was 12.84. The DMFT number in the deciduous teeth was lower in urban patients (5.0) than from rural patients (5.4). The DMFT number in the permanent teeth was lower in urban patients (3.155) than rural patients (3.98). There was no statistically significant correlation between the DMFT value and the number of Entamoeba gingivalis.

Conclusions. 1) Entamoeba gingivalis occurs in the oral cavity of children. 2) In the presented survey, both DMFT and dmft indexes were high which proves high activity of dental carious. 3) There was no significant statistical correlation observed between dental caries and the presence of Entamoeba gingivalis.
\end{abstract}

\section{Key words}

children, dental caries, Entamoeba gingivalis

\section{INTRODUCTION}

The human oral cavity hosts microorganisms - bacteria, fungi, viruses and protozoa. Entamoeba gingivalis is a cosmopolitan amoebae that is present in the human oral cavity. It may be found on the surface of teeth and gingival, in interdental spaces, carious lesions, gingival pockets and in dental plaque. The infective forms are trophozoites [1]. E. gingivalis has been known to additionally inhabit the tonsillar crypts and the bronchial mucus. $95 \%$ of people with gum disease and $50 \%$ of people with healthy gums are hosts to Entamoeba gingivalis. The cyst formation is not present; therefore, transmission is directly from one person to another by kissing, droplet spray or by sharing eating utensils.

Address for correspondence: Agnieszka Skawińska-Bednarczyk, Chair and Department of Pediatric Dentistry, Medical University of Lublin, Karmelicka 7, 20-081 Lublin, Poland

E-mail: agnieszka.skawinska@gmail.com

Received: 24.05.2017; accepted: 16.11.2017; first published: 28.12.2017
Entamoeba gingivalis may be also found in contaminated water and food. Only the trophozoites are formed and the size is usually 10 micrometer to 20 micrometer in diameter. The Entamoeba gingivalis has pseudopodia that allow them to move quickly. Their spheroid nucleus is 2 micrometer to 4 micrometer in diameter and contains a small central endosome. There are numerous food vacuoles containing cellular debris, blood cells and bacteria [2].

The pathogenicity of protozoa in the oral cavity is not completely understood. The presence of Entamoeba gingivalis in the oral cavity of patients with a good state of the immune system usually does not cause any pathological changes [2]. Factors which are considered to be important, and which affect the pathogenicity of Entamoeba gingivalis, are: age, oral hygiene and dental caries [1]. Fighting these protozoa is possible by maintaining a proper oral hygiene and daily treatments. 


\section{OBJECTIVES}

The aim of the study was to evaluate the prevalence of Entamoeba gingivalis among children treated in the Department of Pediatric Dentistry of the Medical University in Lublin and its correlation with dental caries.

\section{MATERIALS AND METHOD}

Diagnostic materials in the form of swabs were obtained from 154 children aged 2-18, treated in the Department of Pediatric Dentistry of the Medical University in Lublin. The main index for epidemiological surveys in oral health - DMFT (decayed, missed, and filled teeth) for permanent teeth and $\mathrm{dmft}$ for deciduous teeth was calculated.

Specimens taken from the gingival pouch, subgingival areas and gums by using sterile swabs were examined for the presence of Entamoeba gingivalis. The trophozoites were noticed in microscopic preparations (Microscope CX41, Olympus, Tokyo, Japan; 400×) 30 minutes after sampling.

The swabs were washed in $0.9 \% \mathrm{NaCl}$ solution. A drop of each sample was placed under a microscope (type, company, town, country) and $0.1 \%$ safranin aqueous solution placed on the slide in order to make the parasites' cell morphology visible and facilitate counting (Fig. 1). Simultaneously, the samples were stained with trichrome according to Wheatley's method for further light microscopic studies (160x and 1000x).

Entamoeba gingivalis cells were identified by observation of morphology and characteristic movement of pseudopodia.

The obtained study results were statistically analyzed using SPSS Statistics 21 (IBM, Warsaw, Poland).

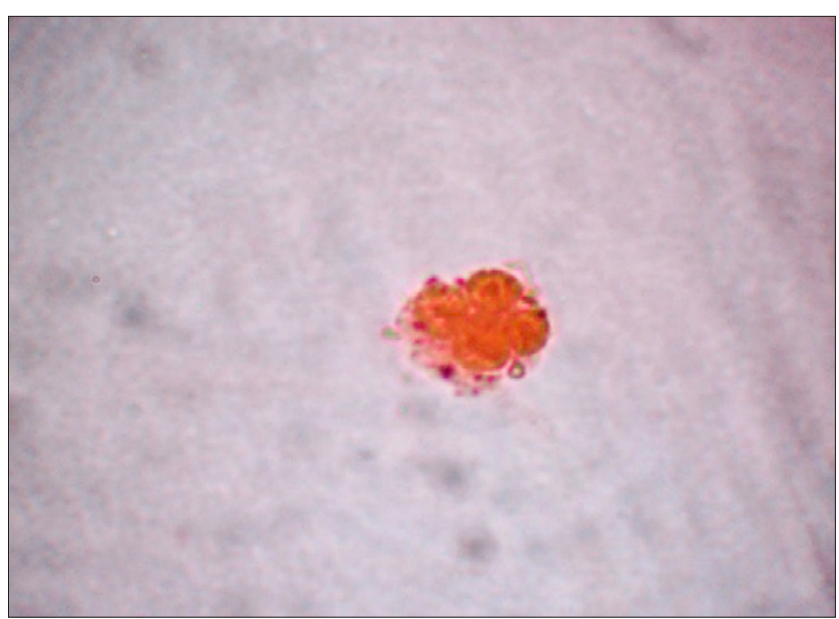

Figure 1. Trophozoite of Entamoeba gingivalis on a direct smear from the oral cavity, coloured with safranine. Magn. 1,000x

\section{RESULTS}

The average number of Entamoeba gingivalis in urban children was 12.84 unity. A lower number of amoeba was found in rural children (10.74 unity) (Fig. 2). The value in Mann Whitney U test was 0.728 , level of significance -0.05 .

More amoebas were found in girls (14.24) compared to boys (10.03) (Fig. 3). Value in Mann Whitney U test - 0.295, level of significance -0.05 .

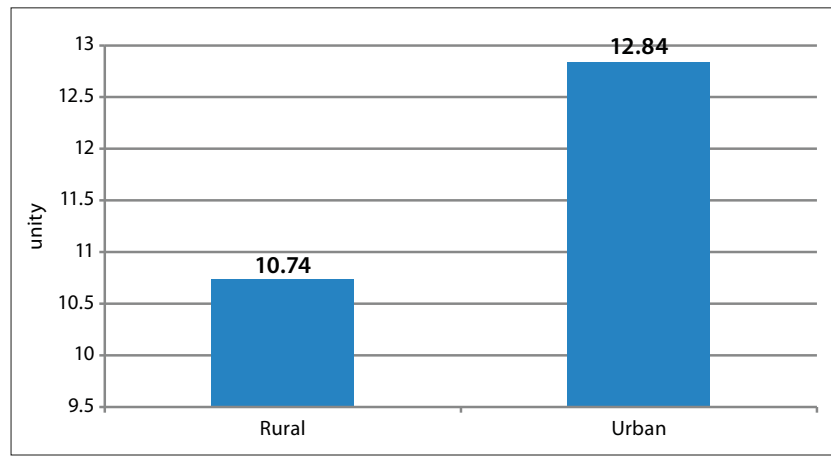

Figure 2. Average number of Entamoeba gingivalis - urban/rural

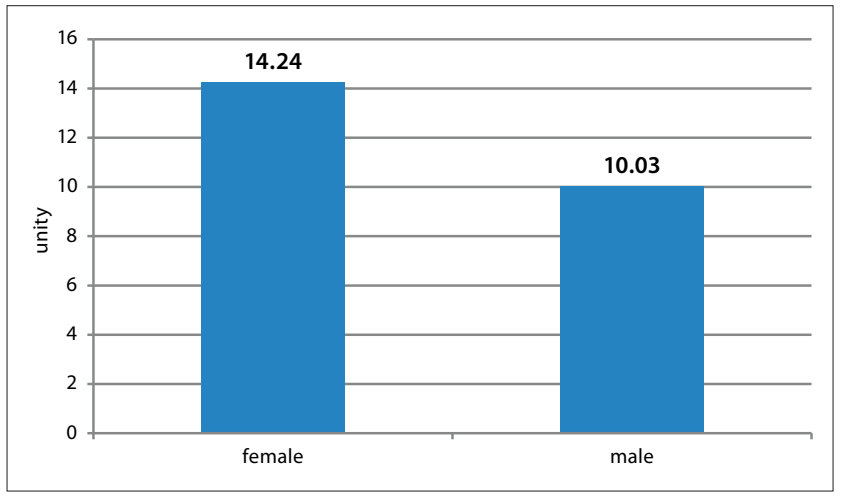

Figure 3. Average number of Entamoeba gingivalis - male/female

The dmft number in the deciduous teeth was lower in urban patients (5.0) than rural patients (5.4). The $\mathrm{dmft}$ in the temporary dentition had the same value in boys and girls. The highest value was seen in the group of patients aged $2-6$ years $(8.6)$.

Pearson's correlation test did not confirm a statistically significant relation between the $\mathrm{dmft}$ value and the number of Entamoeba gingivalis.

The DMFT number in the permanent teeth was lower in urban patients cities (3.155) than from villages (3.98). The DMFT was also lower in boys than girls. The highest value of DMFT was in the group of patients of the age of 11 years and older (9).

Pearson's correlation test did not confirm a statistically significant relation between the DMFT value and the number of Entamoeba gingivalis.

\section{DISCUSSION}

The study by Onyido et al. indicated that individuals with dental diseases and problems are more prone to oral protozoa colonization [4]. This, and previous studies, had similarly associated high oral protozoa harborage in individuals with poor oral and dental hygiene [5].

Vrablic et al. indicated that E. gingivalis does not usually occur among small children and the elderly [6]. In the presented survey, Egingivalis occurred in a group of children. The average number of $E$ gingivalis in urban children was 12.84, whereas a smaller amount of amoebae was found in rural children (10.74). According to some authors, E gingivalis does not occur among small children and its rate of occurrence increases with age [6]. 
Entamoeba gingivalis is transmitted from an infected person and through infected items [7]. Higher levels of amoeba in urban children than in rural children can be explained by the frequency of staying in larger groups, and contacts with a lot of children. In cities there is a greater microbiological contamination [8].

According to Marcinkowska et al., the place of residence has significant impact on the frequency and type of hygiene procedures performed in the oral cavity. Urban children more often clean their teeth and use additional means for dental hygiene [9]. Some authors claim that the use of additional mechanical products for plaque removal, such as dental floss, toothpicks or brushes for cleaning interdental spaces, increases the occurrence of amoebae [10]. This could explain the presented finding that more amoeba were found in uban children.

The presented results also show that the dmft number in deciduous teeth was lower in urban patients (5.0) than from rural patients (5.4). The DMFT in the permanent teeth was lower in urban patients (3.155) than rural patients (3.98).

According to Rodakowska et al., in 2003 the mean number of DMFT in the Podlaskie Region in northeastern Poland was 4.76 in children aged 12 years. In 2003, the value of DMFT was 5.08 in rural areas and 4.42 in urban areas.. In 2010, the difference in favour of 12-year-old children from urban areas was significant and DMFT reached 4.77 in rural areas and 2.82 in urban areas [11]. According to the presented survey, a higher rate of dental caries was found in children living in rural areas, which is consistent with studies by other Polish authors $[10,11]$.

Based on the research, it can therefore be stated that there are still inequalities in access to dental care. In Poland, the inequalities depend on the degree of socio-economic and cultural development of the population [10]. The focus of the Council of the European Union has been to reduce health inequalities, not only at the international level, but also between urban and rural areas [10]. In Scandinavia, New England (USA) and Scotland (UK) dental health was significantly poorer in children living in urban areas compared to those living in rural areas. This might be related to urbanization and a more cariogenic diet; however, within a specific area, the dental health status may differ between sub-groups [12]. In Poland, the environmental urban/rural difference in the intensity of dental caries is less than it was thirty years ago, and does not exceed the threshold of statistical significance [12].

The presented study did not confirm a statistically significant relationship between the DMFT value and the number of Entamoeba gingivalis. According to studies by other authors, the presence of protozoa may be established both in persons with pathological alterations in the oral cavity, and in those with no such symptoms [1]. Abdul- Aziz Kadir et al. found that E. gingivalis occurred among patients with dental caries but was not detected in patients without pathological changes [13], however, the pathogenicity of Entamoeba gingivalis has not been precisely determined [1].

\section{CONCLUSIONS}

An increased incidence of Entamoeba gingivalis in conjunction with pathological changes in the oral cavity was found, particularly among patients with systemic disease, in immunosuppression, after radiotherapy and genetic diseases $[2,3]$. Entamoeba gingivalis occurs in the oral cavity of children. In the presented survey, both DMFT indexes were high which proves high activity of dental carious. There was no significant statistical correlation observed between dental caries and the presence of Entamoeba gingivalis.

\section{REFERENCES}

1. Derda M, Hadaś E, Antczak E, Wojt WJ. Incidence of Entamoeba gingivalis in the oral cavity of students. J Stoma. 2011; 64(10): 784-795.

2. Braz-Silva PH, Magalhães MH CG, Hofman V, Ortega KL, Ilie MI, Odin G, Vielh P, Hofman P. Usefulness of oral cytopathology in the diagnosis of infectious diseases. Cytopathology. 2010; 21(5): 285-289.

3. Zhu J, Zhu M, Yang SG, Wang SJ, Wei RH. Entamoeba gingivalis infection among the students in Shiyan. J Yunyang Med College. 2010; 29(3): 12-13.

4. Onyido AE, Amadi ES, Olofin I, Onwumma AA, Okoh IC, Chikwendu CI. Prevalence of Entamoeba gingivalis and Trichomonas tenax among dental patients attending Federal School of Dental Technology and Therapy clinic, Enugu, Nigeria. Nature and Science. 2011; 9(9): 59-62.

5. Talaro KP, Talaro A. Foudation in Microbiology. 4th Ed. McGraw Hill Companies Inc New York: 2002: 697-19.

6. Vráblic J, Tomová S, Catár G. Occurrence of the protozoa, Entamoeba gingivalis and Trichomonas tenax in the mouths of children and adolescents with hyperplastic gingivitis caused by phenytoin. Bratisl Lek Listy. 1992; 93(3): 136-40.

7. Chomicz L, Piekarczyk J, Zawadzki P, Siemińska- Piekarczyk B, Perkowski K, Piekarczyk P, Szałwiński M. Amoabae transmitted into the human oral cavity and their role in the development of pathological changes - on the basis of literature. Czas stomatal. 2007; 60(5): 321-329.

8. Nooraldeen K. Contamination of public squares and parks with parasites in Erbil city, Iraq. Ann Agric Environ Med. 2015; 22(3): 418-420.

9. Yentur Doni N, Gürses G, Şimşek Z, Yıldız Zeyrek F. Prevalence and associated risk factors of intestinal parasites among children of farm workers in the southeastern Anatolian region of Turkey. Ann Agric Environ Med. 2015; 22(3): 438-442.

10. Gaszyńska E, Wierzbicka M, Marczak M, Szatko F. Thirty years of evolution of oral health behaviours and dental caries in urban and rural areas in Poland. Ann Agric Environ Med. 2014; 21(3): 557-561.

11. Rodakowska E, Wilczyńska-Borawska M, Bagińska J, Stokowska E. Epidemiological analysis of dental caries In 12-year-old children residing In Urban and rural settings In the Podlaskie region of Northeastern Poland. Ann Agric Environ Med. 2013; 20(2): 325-328.

12. Kramer AA, Hakeberg M, Petzold M, Östberg AL. Demographic factors and dental health of Swedish children and adolescents. Acta Odontol Scand. 2015; 1: 1-8.

13. Abdul- Aziz Kadir M, Al- Mashhadani AM, Tahir DIM. SS, Chapook GO. Prevalence of Trichomonas tenax and Entamoeba gingivalis among patients attending dental clinics in Kirkuk City. Al- Taqani. 2007; 20(1): 36-40. 